\title{
Doença arterial periférica
}

Maria José Ferreira, ${ }^{*}$ Pedro Barroso, ${ }^{* *}$ Nádia Duarte***

\section{RESUMO}

A doença arterial periférica é causada, na maioria dos casos, por aterosclerose, que leva ao desenvolvimento de estenoses e oclusões em artérias major da circulação dos membros inferiores.

A sua manifestação mais frequente é a claudicação intermitente, que é caracterizada por desconforto muscular no membro inferior, produzido pelo exercício, e que alivia com o repouso.

A história clínica e o exame físico, complementado pela medição do índice sistólico, permitem fazer o diagnóstico desta entidade.

Por outro lado, a doença arterial periférica é um marcador de risco cardiovascular, pelo que o aspecto mais importante da terapêutica é a correcção dos factores de risco para aterosclerose.

Deste modo, o papel do médico de família é fundamental, não só no correcto diagnóstico e encaminhamento do doente, mas, essencialmente, na detecção e controle dos factores de risco para aterosclerose. Esta intervenção permite melhorar o prognóstico, não apenas do membro, mas, sobretudo, vital, ao diminuir o risco cardiovascular global.

Palavras-chave: Claudicação Intermitente; Aterosclerose; Índice Tornozelo-Braço.

\section{INTRODUÇÃO}

A doença arterial periférica (DAP) é causada, na maioria dos casos, por aterosclerose, que leva ao desenvolvimento de estenoses e oclusões em artérias major da circulação dos membros inferiores.

A sua manifestação mais frequente é a claudicação intermitente, que é caracterizada por desconforto muscular no membro inferior, produzido pelo exercício, e que alivia com o repouso. A claudicação tem um impacto negativo na qualidade de vida dos doentes, quer a nível profissional, quer interferindo com as suas ac-

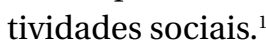

Uma vez que a sua causa mais frequente é a aterosclerose, a correcção dos factores de risco, em fase precoce da doença, pode controlar a sua progressão. ${ }^{2,3,4}$

Por outro lado, a DAP é um marcador de aterosclerose generalizada. Estes doentes têm um elevado risco de mortalidade cardiovascular, de aproximadamente $12 \%$ ao ano, superior aos indivíduos da mesma idade e sexo. ${ }^{5,6}$ Uma vez que o tratamento desta patologia passa mais pela correcção e intervenção nos factores de risco para doença cardiovascular, do que pela intervenção

*Assistente Graduada, Directora do Serviço de Angiologia e Cirurgia Vascular do Hospital Garcia de Orta

**Assistente Hospitalar de Angiologia e Cirurgia Vascular do Hospital Garcia de Orta

***Interna de Angiologia e Cirurgia Vascular do Hospital Garcia de Orta específica a nível da isquemia local, o médico de família tem um papel importante na abordagem destes doentes.

As Sociedades Americana e Europeia de Angiologia e Cirurgia Vascular associaram-se para elaborar um documento de consenso (TASC II - Inter-Society Consensus for the Management of $P A D$ ) sobre a abordagem da DAP. Este documento foi criado em resultado da necessidade de criar guidelines para este problema de saúde pública, e ainda, com o objectivo de orientar os cuidados de saúde primários na gestão destes doentes. Todas as recomendações são apoiadas em níveis definidos de evidência e está disponível, como documento aberto, no endereço www.tasc-2-pad.org. ${ }^{1}$

\section{EPIDEMIOLOGIA}

Estima-se que a prevalência da DAP seja de 3-10 \%. O seu principal sintoma é a claudicação intermitente, cuja prevalência é de cerca de $3 \%$ nos doentes de 40 anos, subindo para $6 \%$ nos doentes sexagenários. Prevê-se que esta prevalência aumente drasticamente nos próximos 20 anos, com o aumento da esperança de

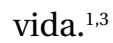

Nos Estados Unidos da América e na Europa efectuaram-se estudos de prevalência da DAP na população geral, em que foi utilizada a determinação do índice tornozelo-braço (ITB) como método de triagem. 
Com este teste verificou-se que, para cada doente com claudicação intermitente, existiam três a quatro com deterioração do ITB, mas que não referiam sintomas., ${ }^{3,7}$ Este facto pode ser explicado por estes doentes se encontrarem em estádios precoces da doença e terem boa compensação colateral, mas, também, pelo estilo de vida, baseado no automóvel e elevador, que não permite que os sintomas se evidenciem. ${ }^{3}$

Em Portugal, a Sociedade Portuguesa de Angiologia e Cirurgia Vascular efectuou, em 2008, um estudo de prevalência da DAP através da determinação do ITB, tendo incluído 5731 indivíduos. Este estudo revelou uma prevalência de 5,9\% de valores de ITB inferiores a 0,9 na população estudada, considerando-se normal um ITB de $1 .^{8}$

As situações de isquemia mais grave, isquemia crítica, são muito menos frequentes. Estima-se que esta seja a forma de apresentação de 1 a $3 \%$ dos doentes com DAP e que apenas 5 a $10 \%$ dos doentes com claudicação intermitente, ou assintomáticos, evoluam para estes estádios, desde que se interrompam os factores de risco cardiovascular. ${ }^{1,4}$

\section{FACTORES DE RISCO}

Os factores de risco para a DAP são comuns à aterosclerose, embora alguns, como o tabagismo e a diabetes, estejam mais implicados no desenvolvimento de lesões na parede das artérias dos membros inferiores. ${ }^{3}$

Os factores de risco não modificáveis incluem a raça, o sexo e a idade. Nos estudos efectuados envolvendo vários países africanos e os Estados Unidos da América, a DAP foi mais frequente nos negros $(7,8 \%$ de prevalência) quando comparados com os caucasianos $(4,4$ $\%$ ), independentemente dos outros factores de risco. ${ }^{3,9}$

Nestes indivíduos observa-se uma maior prevalência de arterite afectando as artérias distais, facto provavelmente atribuível a factores genéticos que ainda não estão completamente esclarecidos. ${ }^{3,9}$

Conforme descrito anteriormente, existe uma nítida correlação entre a idade e a prevalência da doença, o que está associado ao envelhecimento da parede arterial. A prevalência da claudicação intermitente é aproximadamente de $3 \%$ nos doentes com 40 anos e sobe para cerca de $6 \%$ em indivíduos com idade superior a 60 anos. 'Também o sexo masculino apresenta maior incidência que o feminino, sobretudo nas idades mais jo- vens, onde a doença pode ser até duas vezes mais frequente. $^{1}$

A relação entre o tabagismo e a DAP foi demonstrada, de forma inequívoca, em 1911, e existe relação entre a gravidade da doença e o número de cigarros consumidos. Os fumadores têm um risco quatro vezes superior de desenvolverem DAP, em comparação com os não fumadores. ${ }^{1}$

A claudicação intermitente é cerca de duas vezes mais frequente nos diabéticos, assim como a necessidade de amputação por isquemia, que é cinco a dez vezes superior. ${ }^{1,10}$

A hipertensão, dislipidémia, hiperhomocisteinémia e a insuficiência renal crónica, são outros factores de risco importantes. ${ }^{11}$

\section{MANIFESTAÇÕES CLÍNICAS}

O sintoma mais frequente da DAP é a claudicação intermitente, que se caracteriza por dor muscular desencadeada pelo exercício (marcha rápida, em plano inclinado ascendente ou maior distância) e que alivia com o repouso. Este padrão deve-se ao facto de a estenose arterial impedir o aumento do suprimento de sangue e oxigénio ao músculo nas situações em que o esforço induz a necessidade de maior aporte. Estas queixas não aparecem em repouso, pois o fluxo é adequado para esta situação. ${ }^{1,12}$

O grupo muscular mais frequentemente afectado é o gemelar, já que a zona arterial mais susceptível à aterosclerose é o sector femoro-popliteu. Noutros casos, a dor localiza-se aos músculos glúteos e das coxas, correspondendo a obstruções aorto-ilíacas, que, frequentemente, se acompanham de impotência sexual nos homens. ${ }^{10} \mathrm{~A}$ impotência vascular caracteriza-se por o doente conseguir obter erecção, mas não conseguir manter o acto sexual até ao fim, sobretudo quando se encontra em posição de missionário, porque o esforço exercido pelos músculos glúteos desvia o fluxo sanguíneo da zona genital. A causa vascular deve ser sempre excluída na abordagem diagnóstica da disfunção eréctil, embora, por vezes e sobretudo nos diabéticos, possam coexistir outras causas, como a neurogénica. ${ }^{10}$

A claudicação intermitente caracteriza-se por permitir um perímetro de marcha estereotipado para um doente individualmente, mas que pode ser variável para doentes com o mesmo padrão de lesões obstruc- 
tivas, o que está relacionado com a capacidade de compensação individual. Esta consiste em algum grau de dilatação arterial, mas o mecanismo essencial de compensação é o desenvolvimento de circulação colateral, fomentada, principalmente, pelo exercício físico e prejudicada pelo sedentarismo. ${ }^{1,12}$

A gravidade da doença é caracterizada pelas limitações que causa na vida diária do doente. Se ela só se manifesta com esforços maiores do que os quotidianos, não é considerada incapacitante; quando impede as tarefas diárias, é considerada incapacitante. Devem, ainda, considerar-se as limitações sobre as actividades profissional e dos tempos livres, assim como sobre aspectos potencialmente mais exigentes da vida diária, como os resultantes de morar em ruas em plano inclinado ou em edifícios sem elevador. ${ }^{1,10}$

Em fases mais graves da doença observam-se queixas de «dor em repouso», localizada aos dedos do pé ou

\begin{tabular}{|c|c|}
\hline \multicolumn{2}{|c|}{$\begin{array}{l}\text { QUADRO I. Classificação da isquemia de Leriche- } \\
\text {-Fontaine }^{1}\end{array}$} \\
\hline Estádios & \\
\hline Grau I & $\begin{array}{l}\text { Assintomático, mas com índice tornozelo-braço } \\
<0,9\end{array}$ \\
\hline Grau II-A & Claudicação intermitente não incapacitante \\
\hline Grau II-B & Claudicação intermitente incapacitante \\
\hline Grau III & Dor em repouso \\
\hline Grau IV & Lesões tróficas \\
\hline
\end{tabular}

\begin{tabular}{|ll}
\hline QUADRO II. Níveis de evidência \\
Grau & Recomendação \\
\hline A & $\begin{array}{l}\text { Baseada em, pelo menos, um estudo clínico } \\
\text { randomizado, controlado de boa qualidade e que } \\
\text { oriente recomendação específica }\end{array}$ \\
\hline B & $\begin{array}{l}\text { Baseado em estudos clínicos bem conduzidos, em } \\
\text { estudos clínicos randomizados de qualidade } \\
\text { inferior ao nível A }\end{array}$ \\
\hline C & $\begin{array}{l}\text { Baseado na evidência de comités de peritos, } \\
\text { opiniões e/ou estudos clínicos de autoridades }\end{array}$ \\
\hline
\end{tabular}

Adaptado de United States Department of Health and Human Services. Agency for Health Care Policy and Research (1993) antepé. Aparece após algum tempo em repouso, e alivia com a pendência, pelo que o doente tem de deambular alguns períodos durante a noite ou dormir com o pé pendente. Esta dor é particularmente resistente aos analgésicos e traduz o sofrimento isquémico dos tecidos, mesmo em repouso. Aumenta caracteristicamente com o decúbito, pois perde-se a acção da gravidade que ajuda na irrigação ao pé. No estádio de maior gravidade aparecem as lesões tróficas, como as úlceras isquémicas e a necrose dos dedos, mantendo-se, frequentemente, um quadro álgico significativo. ${ }^{1,2}$

Estes dois últimos estádios são denominados de isquemia crítica, nome que traduz a gravidade da situação, em que existe risco de perda do membro se não for possível intervir em tempo útil.

As úlceras de origem arterial caracterizam-se por se localizarem sobretudo a nível dos dedos dos pés, serem particularmente dolorosas, pálidas ou com aspectos de necrose, pouco sangrantes, secas e com atrofia da pele dos bordos. ${ }^{1}$

A localização mais frequente é nos dedos, mas poderão estar localizadas a nível das pernas, nos maléolos ou região pré-tibial. Por esta razão, é mandatório palpar os pulsos distais em todas as situações de úlcera, para despiste de compromisso arterial isolado ou concomitante com a insuficiência venosa (úlceras mistas). ${ }^{2}$

Leriche-Fontaine criou uma classificação de quatro estádios da doença arterial periférica, que se encontram descritos no Quadro II.

\section{DIAGNÓSTICO}

A cirurgia vascular é uma especialidade essencialmente semiológica, pelo que uma história clínica circunstanciada e um exame físico simples podem caracterizar a situação.

Os aspectos essenciais do exame físico incluem a observação dos pés, para excluir lesões ou ulcerações, devendo ser pesquisados os espaços interdigitais. Nos estádios de isquemia grave observa-se a eritrose ou eritrocianose, cuja tonalidade varia com a posição, podendo chegar ao «pé de lagosta» nas situações mais graves. ${ }^{1}$

Também característico é o teste de Buerger, ou seja, o pé fica mais pálido com a sua elevação a 30 graus, sendo mais fácil de valorizar na doença unilateral, por com- 
paração com o outro membro. O tempo de recuperação, que deve ser de um a dois segundos, dá uma noção da gravidade da situação. ${ }^{1}$

A palpação dos pulsos (aórtico, femoral, popliteu, pedioso e tibial posterior), quando estes estão presentes, ajuda a excluir doença significativa. Se ausentes, permite localizar a obstrução. A palpação de pulso aórtico permite avaliar a existência de aneurisma da aorta concomitante. ${ }^{1,10}$

A auscultação de sopros femorais está associada a estenoses ilíacas ou femorais.

Um diagnóstico preciso pode ser obtido através da medição do índice sistólico ou medição do índice tornozelo-braço. Este teste consiste na determinação da pressão arterial sistólica, medida no tornozelo, que é dividida pela pressão arterial sistólica, medida no membro superior, ambas em decúbito. O valor normal é de 1 ou 1,1; se inferior a 0,9 existe doença obstrutiva. Valores inferiores a 0,5 significam doença significativa. Valores muito elevados, superiores a 1,4, traduzem calcificação vascular importante, com não compressibilidade arterial, e estão associados a doenças como a diabetes e a insuficiência renal crónica. ${ }^{13}$

Quando a clínica é muito sugestiva de doença arterial periférica, mas a medição do ITB é de 1, pode aumentar-se a sensibilidade deste teste fazendo uma nova medição após período de esforço, como a marcha ou a elevação repetida dos calcanhares. Uma diminuição do índice em 15 a $20 \%$ é indicativa de doença arterial periférica. ${ }^{13}$

O ITB tem, ainda, o potencial de incrementar o scorede estratificação de risco intermédio de Framingham em cerca de 10-20\%. Um ITB anormal neste grupo converte o seu nível de risco cardiovascular para alto risco e, implica, assim, a instituição de medidas de prevenção secundária, já que está associado a alta incidência de doença coronária e cerebrovascular. Um ITB inferior a 0,9 está associado a um aumento de três a seis vezes da mortalidade cardiovascular. ${ }^{5}$

A TASC recomenda a pesquisa de ITB no doente individual (quadro II) em: ${ }^{1}$

- Todos os doentes com sintomas dos membros inferiores com o esforço (recomendação nível evidência B);

- Doentes com idade entre 50-69 anos e factores de risco cardiovascular, particularmente diabetes e taba- gismo (recomendação nível evidência B);

- Todos os doentes com idade superior a 70 anos, independente dos factores de risco (recomendação nível evidência B);

- Todos os doentes com score de risco Framigham 1020 \% (recomendação nível evidência C).

O eco-doppler é um excelente método não invasivo para localizar e definir lesões, quando o objectivo é a decisão de intervenção. Não é, contudo, necessário para o diagnóstico de isquemia, nem para a decisão em relação às medidas terapêuticas iniciais. ${ }^{13}$

Os outros métodos de diagnóstico, como a angiografia, a angio-ressonância e a angiotomografia axial computorizada (angio TC), estão reservados para as situações em que se considera que os doentes beneficiam de terapêutica endovascular ou de cirurgia arterial directa, cujas indicações estão bem definidas, e serão descritas no capítulo da terapêutica. ${ }^{1,2,4,14}$

A angiografia é considerada o "gold-standard» dos testes de imagem, permitindo, ainda, a correcção endovascular das lesões susceptíveis. Se não existe essa perspectiva, o método a seleccionar deve ser o mais disponível e cuja técnica os profissionais melhor dominem. ${ }^{15,16}$

\section{DIAGNÓSTICO DIFERENCIAL}

A claudicação vascular distingue-se da resultante da patologia osteoarticular e da neurológica (compressão radicular) pelo seu padrão característico, muito embora possam coexistir. A claudicação vascular só ocorre após um período de marcha, aliviando com o repouso. Por outro lado, as queixas com origem osteoarticular aparecem quando se inicia a marcha, melhorando posteriormente. $\mathrm{O}$ doente com claudicação vascular não tem queixas musculares em repouso, ao contrário do doente com compressão radicular lombar, cujas queixas se agravam, frequentemente, com o decúbito.

\section{EVOLUÇÃO DA DOENÇA ARTERIAL PERIFÉRICA}

Apesar de a amputação ser a situação mais temível associada à DAP, esta é uma consequência pouco provável. De facto, só 5 a $10 \%$ dos doentes com DAP vêm a evoluir para isquemia crítica aos cinco anos, podendo alguns destes ainda ser revascularizados; só uma pequena percentagem vem a necessitar de amputação de membro (1-3,3\%). ${ }^{5}$ Também apenas 10 a $20 \%$ dos doen- 
tes revelam agravamento da claudicação aos cinco anos. ${ }^{1}$ Esta evolução está directamente dependente do nível de actividade do indivíduo, como já foi salientado.

Verificou-se que 40 a $60 \%$ dos doentes com DAP têm, simultaneamente, doença coronária e cerebrovascular. Também se observa um risco aumentado de eventos cardiovasculares associados à maior gravidade de $\mathrm{DAP}^{2,3,6}$

Mais frequentes são os eventos cardiovasculares, que incluem o enfarte agudo do miocárdio ou o acidente vascular cerebral, com uma mortalidade global, por qualquer das causas, de 10 a $15 \%$ aos cinco anos. ${ }^{13}$

A taxa anual descrita de eventos cardiovasculares major, que incluem enfarte miocárdio, acidente vascular cerebral ou morte de causa vascular, é de aproximadamente $5-7 \%$. A doença coronária é a causa de morte mais comum nestes doentes ( 40 a $60 \%$ ) e a cerebrovascular é responsável por 10 a $20 \%$ das mortes. ${ }^{2,3,6}$

\section{TERAPÊUTICA}

A terapêutica da doença arterial periférica pode ser genericamente dividida em dois grandes grupos. O primeiro engloba as medidas preventivas e correctivas dos factores de risco e o segundo a terapêutica farmacológica e cirúrgica.

1. Modificar ou eliminar factores de risco cardiovascular: as recomendações da TASC foram baseadas essencialmente em grandes estudos efectuados em doentes com doença coronária e extrapolados para a população com DAP. ${ }^{1,17}$

\begin{tabular}{|c|c|}
\hline Quadro III. Trein & de marcha supervisionado ${ }^{1,2}$ \\
\hline Frequência & 3-5 sessões/ semana \\
\hline Duração & 35-50 m de exercício/sessão \\
\hline Tipo de exercício & $\begin{array}{l}\text { Tapete rolante ou em pista até quase à } \\
\text { dor máxima da claudicação }\end{array}$ \\
\hline Quanto tempo & Mais de 6 meses \\
\hline Resultados & $\begin{array}{l}100 \text { a } 150 \% \text { melhoria do perímetro de } \\
\text { marcha e melhoria associada da } \\
\text { qualidade de vida }\end{array}$ \\
\hline
\end{tabular}

- Cessação tabágica

- Controlo da diabetes mellitus (hemoglobina glicosilada $<7.0 \%$ )

- Controlo da dislipidémia (LDL $<100 \mathrm{mg} / \mathrm{dl}$ )

- Controlo de hipertensão (TA < 140/90 mmHg e 130/80, se diabéticos)

Neste grupo, podemos, ainda, incluir o treino de marcha. Está demonstrado que a prática de exercício físico regular, pelo menos três vezes por semana, aumenta o perímetro total da marcha e a capacidade máxima de exercício em doentes com manifestações de aterosclerose nos membros inferiores (grau IIB da classificação de Leriche-Fontaine). ${ }^{1,12,18}$

Os programas mais eficazes incluem o uso de passadeira rolante com intensidade suficiente para desencadear queixas de claudicação, seguido de período de repouso, em sessões de 30-60 minutos/dia, cerca de três vezes por semana, durante um período de cerca de três meses. (Quadro III) ${ }^{2}$

2. Terapêutica farmacológica e cirúrgica

2.1 Terapêutica farmacológica

A terapêutica farmacológica é transversal a todas as abordagens clínicas no doente com doença aterosclerótica (não exclusivamente dos membros inferiores).

O seu objectivo é diminuir a ocorrência de eventos cardíacos, cerebrais ou isquémicos dos membros inferiores, por prevenção da progressão da doença aterosclerótica.

Os dois grupos farmacológicos mais importantes são os antiagregantes plaquetários e as estatinas, nas doses habituais, pelo seu papel na estabilização da placa aterosclerótica e prevenção de ocorrência de fenómenos de trombose. É importante, também, realçar a terapêutica farmacológica disponível para a correção e controlo dos factores de risco (hipertensão, diabetes mellitus, dislipidémia). Pretendem-se valores de colesterol LDL inferiores a $100 \mathrm{mg} / \mathrm{dl}^{12}$

Fármacos vasodilatadores e hemorreológicos, como a pentoxifilina e o naftidrofuril, têm, também, algum papel na terapêutica dos doentes com claudicação intermitente, embora com resultados controversos nos estudos efectuados. O naftidrofuril está disponível na Europa há mais de 20 anos, tendo efeitos a nível da melhoria do metabolismo do músculo e na redução da agregação eritrocitária e plaquetária. Foram efectuados vários estudos em doentes claudicantes, com a dose 
de $600 \mathrm{mg} /$ dia, verificando-se melhoria clínica quando comparado com o placebo. Os efeitos secundários são ligeiros, sobretudo do foro gastrointestinal. Está, assim, recomendado na TASC, com nível de evidência A. ${ }^{1,19,20}$

A pentoxifilina baixa o nível de fibrinogénio, melhorando, ainda, a deformabilidade dos glóbulos brancos e vermelhos, com efeitos na viscosidade sanguínea. Os resultados dos estudos têm sido QUADRO IV. Resumo das alternativas terapêuticas

\begin{tabular}{l|l|l|l} 
& $\begin{array}{l}\text { Benefício no tapete } \\
\text { rolante/Qualidade } \\
\text { Intervenção }\end{array}$ & Lida & $\begin{array}{l}\text { Percentagem de } \\
\text { doentes com DAP } \\
\text { em que está indicado }\end{array}$ \\
\hline Exercício & $100 \% /$ Melhoria & Disponibilidade & \\
\hline Motivação & $50-85 \%$ & Insuficiência cardíaca & $50-85 \%$ \\
\hline Cilostazol & $50 \% /$ Melhoria & $\begin{array}{l}\text { Melhor nas artérias } \\
\text { proximais (ilíacas) }\end{array}$ & $10-15 \%$ \\
\hline Angioplastia & Melhoria & $\begin{array}{l}\text { Falência de enxerto, } \\
\text { morbilidade, } \\
\text { mortalidade }\end{array}$ & $<5 \%$ \\
\hline Cirurgia & $150 \% / M e l h o r i a$ & & \\
\hline
\end{tabular}
controversos, por não serem completamente reprodutíveis. ${ }^{21}$

As técnicas que podem ser usadas em doentes com

O cilostazol, fármaco ainda não disponível em Portugal, tem revelado maior nível de evidência no tratamento destas situações, demonstrando melhoria da performance no tapete rolante e da qualidade de vida, quando comparado com o placebo. Pertence aos inibidores da fosfodiesterase III, com actividade vasodilatadora, metabólica e antiplaquetária. ${ }^{14}$

\subsection{Terapêutica cirúrgica}

No subgrupo de doentes em que, por mau controlo dos factores de risco ou má adesão terapêutica, ocorre progressão da doença, a vertente interventiva pode ser uma alternativa viável.

Os doentes claudicantes só têm indicação cirúrgica se a claudicação for incapacitante, depois de ter efectuado período de 3 meses com terapêutica adequada: cessação tabágica, treino de marcha, controle da hipertensão, diabetes e hiperlidipidémia, assim como medicação.

Se as queixas se mantiverem incapacitantes, devem ser efectuados exames de imagem para definição da anatomia vascular (angiografia ou angioressonância ou angio TC)

São candidatos a revascularização os doentes com dor em repouso e os com lesões tróficas, ou seja, isquemia crítica, pelo risco eminente de perda de membro. ${ }^{1,2,4,14}$

Os doentes acamados ou em cadeira de rodas, que não deambulam, ou com flexo fixo do joelho têm contra-indicação absoluta para revascularização. .,2,4,13 $^{2}$ doença aterosclerótica dos membros inferiores podem ser divididas em dois grupos:

\section{A. Cirurgia arterial directa}

- Bypass (cirurgia que ultrapassa a lesão arterial utilizando um conduto - veia ou prótese). Por exemplo: bypass femoro-popliteo, bypass ílio-femoral, bypass aorto-bifemoral.

- Endarterectomia (cirurgia em que é removida a placa de ateroma de artéria).

- Tromboembolectomia (acto em que, utilizando um cateter, se remove o coágulo de sangue da artéria).

\section{B. Cirurgia Endovascular}

- Angioplastia

- Colocação de stent

- Colocação de endoprótese

A opção entre a cirurgia ou a terapêutica endovascular depende das condições clínicas do doente, das características e localização das lesões ateroscleróticas, assim como da experiência do centro vascular de referenciação.

A opção pela terapêutica endovascular apresenta a grande vantagem de ser uma técnica menos invasiva e, portanto, com tempos de internamento mais curtos.

Os resultados clínicos disponíveis revelam que as duas abordagens terapêuticas apresentam resultados idênticos em termos de sobrevida do doente e permeabilidade arterial, estando condicionadas pelo tipo e distribuição das lesões. ${ }^{1}$

O Quadro IV resume as alternativas terapêuticas. 


\section{SEGUIMENTO DOS DOENTES}

Numa primeira abordagem após o diagnóstico, o doente deve ser reavaliado aos três meses, para verificar a adesão às medidas instituídas, principalmente o abandono tabágico e o cumprimento do protocolo de marcha. Deve, ainda, ser registado o perímetro de marcha sem claudicação, para comparação com o prévio à terapêutica.

Se o doente aderir à terapêutica, o seguimento posterior deve ser efectuado anualmente. Justifica-se a medição de ITB se existir agravamento clínico significativo (diminuição do perímetro de marcha sem claudicação ou evolução para isquemia crítica). Como é óbvio, esta evolução justifica, ainda, reorientação para consulta de especialidade e, no caso de isquemia crítica, com critério de urgência.

\section{CRITÉRIOS DE REFERENCIAÇÃO}

É fundamental a avaliação precoce dos doentes com patologia arterial aterosclerótica, no sentido da correcção e terapêutica dos factores de risco. É igualmente essencial a orientação atempada dos doentes seleccionados para consulta de especialidade, após uma correcta avaliação clínica, complementada com a determinação do índice tornozelo-braço.

Todos os doentes com sintomatologia de claudicação intermitente incapacitante de etiologia aterosclerótica deverão ser avaliados por Angiologia e Cirurgia Vascular.

Se, apesar do correcto cumprimento da terapêutica conservadora, as queixas de claudicação forem incapacitantes, prejudicando o estilo de vida do doente, este deverá ser encaminhado para consulta de especialidade para avaliação da possibilidade de terapêutica interventiva.

Os doentes com isquemia crítica (estádio de dor em repouso ou com lesões tróficas) devem ser encaminhados com critérios de urgência.

\section{CONCLUSÃO}

A claudicação intermitente de causa vascular é uma situação de relativo bom prognóstico do ponto de vista de viabilidade do membro. Na maioria dos casos, a sua terapêutica é médica, baseada na correcção dos factores de risco para aterosclerose e exercício de marcha. Como foi referido, apenas uma pequena percentagem de doentes vem a necessitar de cirurgia ou a progredir para isquemia crítica e amputação, se deixar de fumar e corrigir os factores de risco para aterosclerose já especificados.

O fundamental desta entidade clínica é o facto de ser um importante marcador de risco cardiovascular e alertar para a necessidade de intervir a esse nível, obrigando a estudos cardíacos (ECG, ecocardiograma) e ecodopler carotídeo, para melhor estratificação de risco, assim como a uma intervenção no sentido da sua modificação.

A claudicação intermitente é uma oportunidade, nalguns doentes, para modificar o estilo de vida, podendo proporcionar, se bem aproveitada, a diminuição dos eventos cardiovasculares incapacitantes ou fatais.

A DAP não é uma patologia da exclusiva responsabilidade e interesse da Cirurgia Vascular. Pela sua frequência, gravidade e implicações pessoais, sociais e económicas, é um importante problema de saúde pública, que corta transversalmente todas as especialidades médicas e cirúrgicas.

Os especialistas de Cirurgia Vascular tratam a «ponta do icebergue». Todos os outros, particularmente os médicos de Medicina Geral e Familiar, fazem prevenção, prospecção, tratamento e acompanhamento da doença, como ficou exposto.

\section{REFERÊNCIAS BIBLIOGRÁFICAS}

1. Norgren L, Hiatt WR, Dormandy JA, Nehler MR, Harris KA; Fowkes FG; TASC II Working Group. Inter-Society Consensus for the Management of Peripheral Arterial Disease (TASC II). J Vasc Surg 2007 Jan; 45 Suppl S: S5-67.

2. Hirsch AT, Criqui MH, Treat-Jacobson D, Regensteiner JG, Creager MA, Olin JW, et al. Peripheral arterial disease detection, awareness, and treatment in primary care. JAMA 2001 Sep 19; 286 (11): 1317-24.

3. Selvin E, Erlinger TP. Prevalence of and risk factors for peripheral arterial disease in the United States: results from the National Health and Nutrition Examination Survey, 1999-2000. Circulation 2004 Aug 10; 110 (6): 738-43.

4. Dormandy JA, Murray GD. The fate of the claudicant: a prospective study of 1969 claudicants. Eur J Vasc Surg 1991 Apr; 20 (2): 384-92.

5. Kannel WB, Skinner JJ Jr, Schwartz MJ, Shurtleff D. Intermittent claudication: Incidence in the Framigham Study. Circulation 1970 May; 41 (5): 875-83.

6. Fowkes FG, Murray GD, Butcher I, Heald CL, Lee RJ, Chambless LE, et al. Ankle brachial index combined with Framingham Risk Score to predict cardiovascular events and mortality: a meta-analysis. JAMA 2008 Jul 9: 300 (2): 197-208.

7. Mourad JJ, Cacoub P, Collet JP, Becker F, Pinel JF, Huet D, et al. Screen- 
ing of unrecognized peripheral arterial disease (PAD) using anklebrachial index in high cardiovascular rick patients free from symptomatic PAD. J Vasc Surg 2009 Sep; 50 (3): 572-80.

8. Menezes JD, Fernandes JF, Carvalho CS, Barbosa J, Mansilha A. Estudo da prevalência da doença arterial periférica em Portugal. Rev Soc Port Angiol Cir Vasc, 2009; 5 (2): 59-68.

9. Kullo IJ, Bailey KR, Kardia SL, Mosley TH Jr, Boerwinckle E, Turner ST. Ethnic differences in peripheral arterial disease in the NHLBI Genetic Epidemiology Network of Artriopathy (GENOA) study. Vasc Med 2003 Nov; 8 (4): 237-42.

10. Brewster DC. Direct reconstruction for aortoiliac occlusive disease. In: Rutherford RB (editor). Vascular Surgery. 6th ed. Philadelphia, PA: Elsevier; 2005

11. Ridker PM, Stampfer MJ, Rifai N. Novel risk factors for systemic atherosclerosis a comparison of C-reactive protein, fibrinogen, homocystein, lipoprotein(a), and standard cholesterol screening as predictors of peripheral arterial disease. JAMA 2001 May 16; 285 (19): 2481-5.

12. Defraigne JO. Development of atherosclerosis for the vascular surgeon. In: Liapis CD, Balzer K, Benedetti-Valentini F, Fernandes JF. Vascular Surgery. Berlin: Springer, 2007. p. 23-34.

13. Rutherford RB, Lowenstein DH, Klein MF. Combining segmental systolic pressures and plethysmography to diagnose arterial occlusive disease of the legs. Am J Surg 1979; 138 (2): 211-8.

14. Regensteiner JG, Ware JE Jr, McCarthy WJ, Zhang P, Forbes WP, Heckman J, et al. Effect of cilostazol on treadmill walking, community-based walking ability, and health-related quality of life in patients with intermittent claudication due to peripheral arterial disease: meta-analysis of six randomized controlled trials. J Am Geriatr Soc 2002 Dec; 50 (12): 1939-46.

15. Ota H, Takase K, Igarashi K, Chiba Y, Haga K, Saito H, et al. MDCT compared with digital subtraction angiography for assessment of lower ex- tremity arterial occlusive disease: importance of reviewing cross-sectional images. AJR Am J Roentgenol 2004 Jan; 182 (1): 201-9.

16. Jakobs TF, Wintersperger BJ, Becker CR. MDCT-imaging of peripheral arterial disease. Semin Ultrasound CT MR 2004 Apr; 25 (2): 145-55.

17. Hiatt W. Atherogenesis and the Medical Management of Atherosclerosis. In: Rutherford RB (editor). Vascular Surgery. 6th ed. Philadelphia, PA: Elsevier; 2005.

18. Stewart KJ, Hiatt WR, Regensteiner JG, et al. Exercise training for claudication. N Engl J Med 2002; 347 (24): 1941-51.

19. Kieffer E, Bahnini A, Mouren X, Gamand S. A new study demonstrates the efficacy of naftidrofuril in the treatment of intermittent claudication. Findings of the Naftidrofuryl Clinical Ischemia Study (NCIS). Int Angiol 2001; 20 (1): 58-65.

20. Spengel F, Clément $D$, Boccalon $H$, Liard F, Brown T, Lehert P. Findings of the Naftidrofuryl in Quality of life (NIQOL) European study program. Int Angiol 2002; 21 (1): 20-7.

21. Hood SC, Moher D, Barber GG. Management of intermittent claudication with pentoxifylline: meta-analysis of randomized controlled trials. CMAJ 1996 Oct 15; 155 (8): 1053-9.

\section{CONFLITOS DE INTERESSE}

Os autores declaram não existirem conflitos de interesse na elaboração deste artigo

\section{ENDEREÇO PARA CORRESPONDÊNCIA}

Pedro Barroso e Maria José Ferreira

Hospital Garcia da Orta, Av. Prof. Torrado da Silva, Pragal, Almada

mjferreira@hgo.min-saude.pt

Recebido em 15/07/2010

Aceite para publicação em 26/10/2010

\section{ABSTRACT}

\section{PERIPHERAL ARTERIAL DISEASE}

Peripheral arterial disease is caused mainly by atherosclerosis, which leads to arterial stenosis and occlusions in the major vessels that supply the lower limbs.

The classic symptom is intermittent claudication, which is characterized by muscle discomfort in the lower limb, caused by exercise and relieved by rest.

An accurate diagnosis of peripheral arterial disease is achieved by obtaining the patient's vascular history and physical examination. The ankle brachial index can complement the evaluation.

Peripheral arterial disease is a marker of cardiovascular risk, so the most important aspect of the therapy is the correction of the risk factors for atherosclerosis.

The Family Doctor thus plays a crucial role, not only on the correct diagnosis and referral of the patient but mainly in the detection and control of the risk factors that lead to atherosclerosis. This intervention reduces the global cardiovascular risk and allows for the improvement of the prognosis, both on the lower limb and of the general condition.

Keywords: Intermittent Claudication; Atherosclerosis; Ankle Brachial Index. 